This is the accepted version of the article:

Heli B., Morales-Narváez E., Golmohammadi H., Ajji A., Merkoçi A.. Modulation of population density and size of silver nanoparticles embedded in bacterial cellulose: Via ammonia exposure: Visual detection of volatile compounds in a piece of plasmonic nanopaper. Nanoscale, (2016). 8. : 7984 - . 10.1039/c6nr00537c.

Available at: https://dx.doi.org/10.1039/c6nr00537c 


\title{
MODULATION OF POPULATION DENSITY AND SIZE OF SILVER NANOPARTICLES EMBEDDED IN BACTERIAL CELLULOSE VIA AMMONIA EXPOSURE: VISUAL DETECTION OF VOLATILE COMPOUNDS IN A PIECE OF PLASMONIC NANOPAPER
}

\author{
B. Heli ${ }^{a b}$, E. Morales-Narváez ${ }^{a}$, H. Golmohammadi ${ }^{c}$, A. Ajji ${ }^{*}{ }^{b}$ and A. Merkoçi $* d$ \\ aCatalan Institute of Nanoscience and Nanotechnology (ICN2), CSIC and The Barcelona Institute of \\ Science and Technology, Campus UAB, Bellaterra, Barcelona 08193, Spain \\ ¿3SPack, CREPEC, Département de génie chimique, Polytechnique Montréal, Montréal, Québec, \\ Canada \\ ‘ACECR-Production Technology Research Institute, Ahvaz, 6139684689, Iran \\ ${ }^{\circledR}$ ICREA - Catalan Institution for Research and Advanced Studies, Barcelona, 08010, Spain.
}

Published in: NANOSCALE, 2016, Vol. 8, Page 7984-7991

DOI: $10.1039 / c 6 n r 00537 c$ 


\subsection{Abstract}

The localized surface plasmon resonance exhibited by noble metal nanoparticles can be sensitively tuned by varying their size and interparticle distances. We report that corrosive vapor (ammonia) exposure dramatically reduces the population density of silver nanoparticles (AgNPs) embedded within bacterial cellulose, leading to a larger distance between the remaining nanoparticles and a decrease in the UV-Vis absorbance associated with the AgNP plasmonic properties. We also found that the size distribution of AgNPs embedded in bacterial cellulose undergoes a reduction in the presence of volatile compounds released during food spoilage, modulating the studied nanoplasmonic properties. In fact, such a plasmonic nanopaper exhibits a change in color from amber to light amber upon the explored corrosive vapor exposure and from amber to a grey or taupe color upon fish or meat spoilage exposure. These phenomena are proposed as a simple visual detection of volatile compounds in a flexible, transparent, permeable and stable single-use nanoplasmonic membrane, which opens the way to innovative approaches and capabilities in gas sensing and smart packaging.

\subsection{Introduction}

Nanomaterials display outstanding and high-powered phenomena that are not present in bulk materials [1], such as localized surface plasmon resonance in noble metal nanoparticles (NPs) [2], where incident light triggers collective motion of their electrons, amplifying the local electromagnetic field. Noble metal NPs are stable, easy to synthesize and, unlike photoluminescent NPs, endowed with a relatively endless amount of photons for sensing applications over long time periods, which can even be visually detected [3-6]. Given these extraordinary behaviors, nanosensing technology is capitalizing on analyte-nanoparticle interactions that modulate the size, shape, composition, and interparticle distance of the NPs, the dielectric properties or the refractive index of the surrounding medium to engineer advantageous nanoplasmonic sensing systems [3,7-10].

Recently, we have proposed bacterial cellulose nanopaper decorated with optically active nanoparticles, including plasmonic NPs and photoluminescent NPs, as a novel sensing platform targeting analytes in the liquid phase [11]. On the other hand, plasmonic NPs have generally been exploited to detect analytes in the liquid phase [3]. However, the detection of biologically relevant analytes in the gas phase using simple platforms based on plasmonic NPs is scarcely explored. For 
example, in early 2016, a search on Web of Science using the formula topic: ((localized surface plasmon resonance) and (gas detection)), produces results pointing to less than 50 articles, none of which are related to visual detection.

Some gaseous analytes, particularly volatile compounds are often associated with environmental pollution, hazardous chemicals and potential biomarkers [12-15]. Furthermore, these agents can also be found as a by-product of bacteria and food spoilage or even in the headspace of cancer cells and blood samples [16-18]. Therefore, the detection of volatile compounds is a paramount research field and related technologies remain under development. On the one hand, emerging approaches intended for volatile compound sensing are expected to be simple, safe, low-power, stable, inexpensive and easy-to-use. On the other hand, commercially available paper tests offer fast and sensitive detection strips to measure dangerous gases in the air. However, they are often hazardous items themselves and/or require a moistening step or external reagents $[19,20]$. Here, we demonstrate a simple-to-use plasmonic nanopaper designed as a hybrid material based on silver nanoparticles embedded in bacterial cellulose that is amenable to the detection of analytes in the gas phase with no extra reagents. In particular, we discovered that corrosive vapor (ammonia) exposure dramatically reduces the population density or size distribution of silver nanoparticles (AgNPs) embedded within the studied nanoplasmonic membrane, leading to a modulation of the UV-Vis absorbance corresponding to the explored nanoplasmonic properties, which can even be determined visually. Error! Reference source not found. conveys the proposed sensing principle and depicts the overall set-up utilized throughout this research, which represents a straightforward way to follow plasmonic nanopaper interaction with gases. 



Scheme 1. A. Schematic representation of the nanoplasmonic membrane upon volatile compound exposure. Corrosive vapor (ammonia) triggers etching of the nanoparticles embedded within the studied nanoplasmonic membrane (a). Therefore, the population density of the AgNPs falls and the interparticle distance increases, and/or the size distribution undergoes a dramatic change, changing the color of the plasmonic nanopaper. B. Schematic representation of the setup utilized throughout this research. A sealed container with a capacity of $50 \mathrm{~mL}$ is used. A piece of plasmonic nanopaper is attached underneath the container lid and vertically aligned through a holder being exposed to a volatile compound, which was previously introduced.

\subsection{Experimental}

\subsubsection{Reagents and equipments}

The commercial reagents were handled according to the material safety data sheets suggested by the suppliers. Bacterial cellulose nanopaper was kindly provided free of charge by Nanonovin Polymer 
Co. (Mazandaran, Iran). Ammonia (30\%), methanol (99.9\%), ethanol (99.8\%), propanol (99.8\%), acetic acid (100\%), ethyl acetate (99\%), acetonitrile (99.8\%), acetone (99.5\%), and toluene (99.8\%) were purchased from Panreac AppliChem (Castellar del Vallès, Barcelona, Spain). $\mathrm{NaOH}, \mathrm{AgNO}_{3}$, indole (99\%), trimethylamine (99.5\%) and microwell plates were acquired from Sigma-Aldrich (Taufkirchen, Germany). The thickness of bare bacterial cellulose (BC) and the nanoplasmonic membrane was respectively measured using a digital calliper (Alfa Mirage Co. Ltd, Osaka, Japan) and reported as an average of 10 different points in various pieces of paper. Particle size distributions and population density were estimated using Image J 1.48v (Wayne Rasband, National Institutes of Health, USA). UV-Vis absorbance spectra were recorded using a SpectraMax M2e spectrophotometer (Molecular Devices, California, USA). SEM imaging was performed using a Magellan 400L SEM High-Resolution SEM (FEI, Oregon, USA). TEM imaging was carried out using a FEI Tecnai F20 S/TEM (Oregon, USA). Nanopaper samples were prepared using a Precision Ion Polishing System, Model 691 with camera, (GATAN, California, USA) before the TEM analysis. Raman spectroscopy was performed using a Renishaw Invia Raman Microscope System (Gloucestershire, UK). The error bars in the figures represent the standard deviation of three parallel experiments. The box plots in Figure 4-1 and Figure 0-4 show the median, 25th and 75th percentiles and the extreme values of the respective size distributions (5-95 percentile). All of the experiments were carried out using the experimental set-up described in eme 1B. 

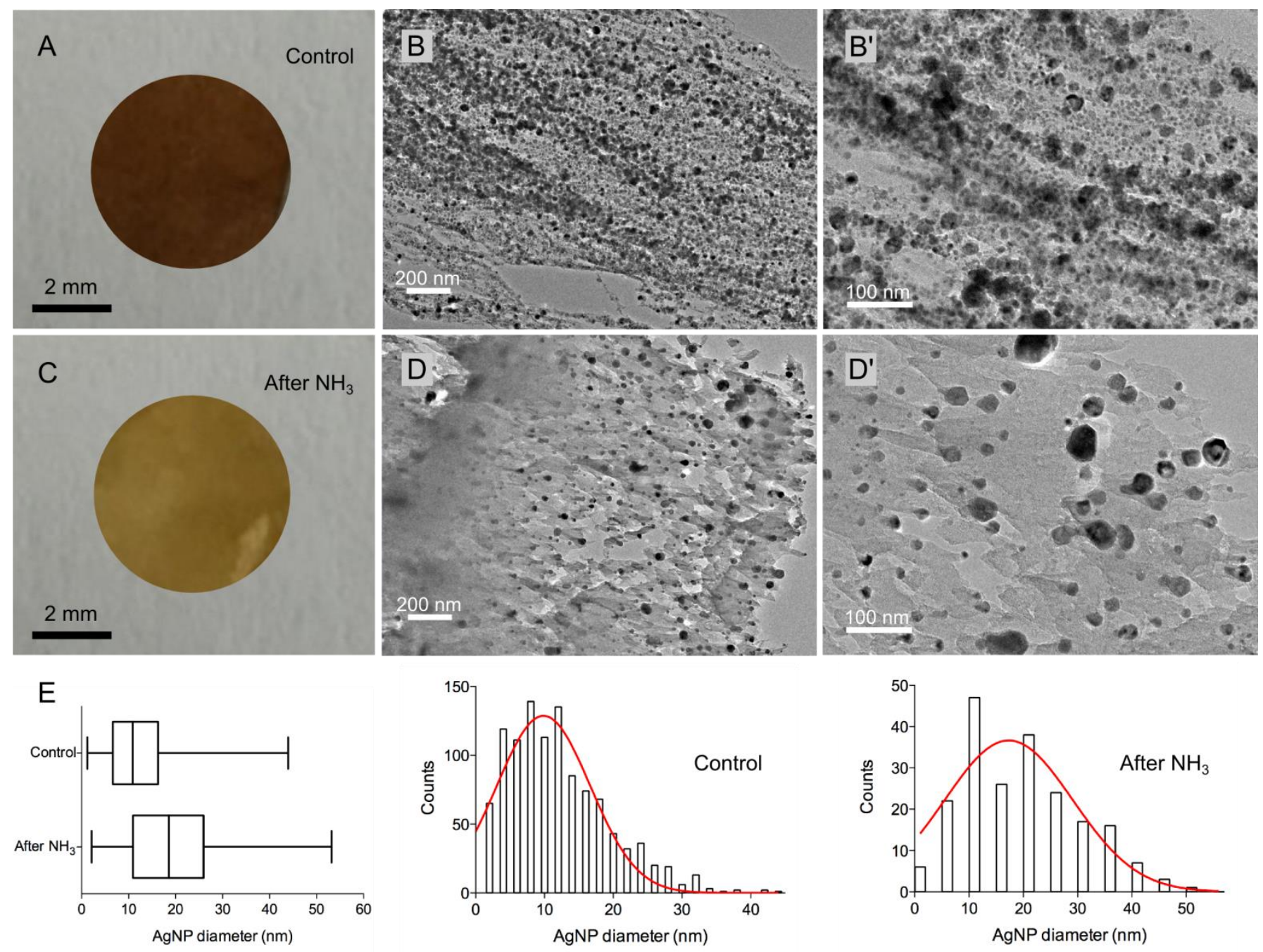

Figure 0-1. Modulation of the population density of AgNPs embedded in bacterial cellulose via corrosive vapor (ammonia) exposure. Appearance of AgNP-BC without ammonia vapor exposure (A) and after ammonia vapour exposure (C). TEM micrographs of AgNP-BC without ammonia vapour exposure (B and $\left.\mathrm{B}^{\prime}\right)$ and after ammonia vapour exposure $\left(\mathrm{D}\right.$ and $\left.\mathrm{D}^{\prime}\right)$. Estimation of the size distribution of the studied AgNPs (E) without ammonia vapour exposure (control) and after ammonia vapour exposure. The box plots show the median, 25th and 75th percentiles and the extreme values of the respective size distributions (5-95 percentile). 


\subsubsection{Synthesis of plasmonic nanopaper}

Silver nanoparticles were synthesized in situ within bacterial cellulose following previous procedures [11,21]. Particularly, 15 pieces of wet bacterial cellulose $(2 \times 2.5 \times 0.3 \mathrm{~cm})$ were soaked in $50 \mathrm{ml}$ of milli-Q water at pH 12 adjusted using $2 \mathrm{M} \mathrm{NaOH}$. Then, the mixture was kept under heating and stirring to reach $65{ }^{\circ} \mathrm{C}$. At this time, $5 \mathrm{ml}$ of a fresh solution of $0.1 \% \mathrm{AgNO}_{3}(\mathrm{v} / \mathrm{w})$ was added to the mixture dropwise. During vigorous stirring at $65^{\circ} \mathrm{C}$, silver ions diffused into the fibril network of the cellulose and gradually converted to silver nanoparticles. After $2 \mathrm{~h}$, silver nanoparticle formation was confirmed by the color change of the colorless bacterial cellulose to amber. Then the heating process was stopped and the mixture was cooled down to room temperature. Bacterial cellulose pieces containing in situ synthesized AgNPs (plasmonic nanopaper pieces) were separated from the mixture and completely washed through the squeezing of BC and redispersing in milli-Q water. This step was repeated 3 times to remove all of the unreacted silver ions and free silver nanoparticles from the cellulose fibrils. The nanoplasmonic membranes were dried at room temperature while they were kept between filter paper and glass slides to prevent any twisting and tearing. The original thickness of bare $\mathrm{BC}$ was not dramatically affected after the synthesis of AgNPs embedded within the $\mathrm{BC}$ as the thickness of the dried nanoplasmonic and BC membranes were estimated to be $16 \pm 1.7$ and $16 \pm 2.5$ $\mu \mathrm{m}$, respectively. Once it is dried, the composite can be stored in the dark at room temperature for more than 6 months. Following the described procedure systematically, we observed that the synthesis of the nanoplasmonic membrane can be reproduced with a similar absorbance spectrum and nanoparticle population.

\subsubsection{Preparation of the gas sensing set-up}

Volatile compound sensing experiments were carried out in sealable containers with a total volume of $50 \mathrm{~mL}$. To explore the gas effect on the nanoplasmonic membrane, tiny amounts of volatile compounds (from 5 to $1000 \mu \mathrm{L}$ ) were drop-casted onto the bottom of the container aiming to spontaneously volatilize the studied compound and expose it to the nanoplasmonic membrane which was at the top of the sealed container (as described in Error! Reference source not found.B). The plasmonic nanopaper pieces were assembled inside a holder made of PDMS films, which was attached underneath the container lid but vertically aligned. Previously, original UV-Vis absorbance spectra of completely dried AgNP-BC pieces $(5 \times 5 \mathrm{~mm})$ were recorded. All of the experiments were carried out in triplicate. 


\subsubsection{Sample preparation for TEM analysis}

The samples made of $\mathrm{BC}$ and nanopaper embedding AgNPs were prepared via three different steps using a Precision Ion Polishing System. (i) The samples were treated with an energy of $4 \mathrm{keV}$ for 30 minutes (angle from the plane of the sample to the incident ion beam 1 of +7 units, and to the incident beam 2 of +7 units). (ii) The samples were treated with an energy of $3 \mathrm{keV}$ for 10 minutes (angle from the plane of the sample to the incident ion beam 1 of +7 units, and to the incident beam 2 of +7 units). (iii) The samples were treated with an energy of $0.5 \mathrm{keV}$ for 5 minutes (angle from the plane of the sample to the incident ion beam 1 of +7 units, and to the incident beam 2 of +7 units).

\subsubsection{Raman analysis}

Raman spectroscopy was carried out using an argon laser of $514 \mathrm{~nm}(25 \mathrm{~mW})$, an objective lens of $50 \times$ and a CCD exposure time of 30 seconds.

\subsection{Results and discussion}

Nanopaper exhibits a myriad of functional properties which are advantageous in optical sensing, including optical transparency [22], sustainability, flexibility [23], high mechanical strength, hydrophilicity, high porosity, broad chemical-modification capabilities and high surface area [2426]. The nanopaper utilized in this research was synthesized via Acetobacter xylinum, as previously described and characterized [11]. Among other features, this cellulose exhibits a crystallinity around $82 \%$, an approximate Young's modulus of $17 \mathrm{GPa}$, an average fiber diameter of $c a .45 \pm 10 \mathrm{~nm}$ and a length that is approximately greater than $10 \mu \mathrm{m}$. The plasmonic nanopaper exploited throughout this research was synthesized following recently reported methods, where AgNPs are synthesized in situ using nanopaper as the reducing agent of a salt-containing $\mathrm{Ag}$ precursor such as $\mathrm{AgNO}_{3}$ [11.21].

\subsubsection{The effect of ammonia vapor on AgNPs embedded in bacterial cellulose nanopaper}

For the present study, we employed ammonia as a model corrosive agent, which is known as an environmental and clinically relevant analyte $[14,15,27]$. In order to explore the effect of corrosive vapor exposure on the nanoplasmonic membrane, $1 \mathrm{~mL}$ of $30 \%$ ammonia solution was introduced and spontaneously volatilized at room temperature for 12 hours using the set-up described in Error! 
Reference source not found.B. Remarkably, the plasmonic nanopaper displayed a change in color from amber to light amber, which can be readily observed by the naked eye when compared with a control piece of plasmonic nanopaper (without ammonia vapor exposure, see Figure 4-1A and C). Once the explored nanopaper samples were analyzed via transmission electron microscopy (TEM), we discovered that the population density of the AgNPs embedded in the nanopaper was dramatically decreased. In fact, the population density of the AgNPs in the images of the control plasmonic nanopaper has been estimated to be $1473 \pm 227 \mathrm{AgNP}_{\mu \mathrm{m}}{ }^{-2}$, whereas the population density of the AgNPs in the images of the plasmonic nanopaper exposed to ammonia in the aforementioned conditions is estimated to be $302 \pm 38 \mathrm{AgNP} \mu \mathrm{m}^{-2}$ (see Figure S0-1). Moreover, the control nanoplasmonic membrane (without ammonia exposure) displays a broad size distribution of AgNPs with an average particle diameter of around $10 \pm 7 \mathrm{~nm}$, whereas the nanoplasmonic membrane exposed to ammonia showed an average particle diameter of around $17 \pm 12 \mathrm{~nm}$ (see Figure 4-1). These results suggest that the AgNPs embedded in the nanopaper are prone to be partially or completely etched by corrosive vapor exposure, leading to a larger distance between the remaining nanoparticles, whereas the average particle size increases, modulating the plasmonic properties of the explored nanoplasmonic membrane. Interestingly, we observed through scanning electron microscopy (SEM) imaging that this phenomenon occurs with virtually no damage to the structure of the nanocellulose (see Figure S0-2).

\subsubsection{Specificity upon ammonia exposure}

We expected that the modulation of the population density of the AgNPs embedded in bacterial cellulose via ammonia exposure would lead to a sensing platform. Aiming at exploring whether the modulation of plasmonic properties discussed above is specific to corrosive vapour (ammonia) exposure, the nanoplasmonic membrane was exposed to other volatile compounds under harsh conditions, including methanol (99.9\%), ethanol (99.8\%), propanol (99.8\%), acetic acid (100\%), ethyl acetate (99\%), acetonitrile (99.8\%), acetone (99.5\%), toluene (99.8\%), indole (99\%) and trimethylamine (99.5\%). Using the set-up described in Error! Reference source not found.B, $1 \mathrm{~mL}$ of each of these volatile compounds was spontaneously volatilized at room temperature for 16 hours, respectively. Likewise, $1 \mathrm{~mL}$ of water was also included in these experiments to evaluate the moisture effect on the plasmonic nanopaper. Afterwards, the UV-Vis absorbance of each piece of plasmonic nanopaper was analyzed. We ascertained that the moisture gave rise to a blue shift of approximately 
$10 \mathrm{~nm}$ in the UV-Vis spectra of the nanoplasmonic membrane, whereas the intensity of the absorbance peak was negligibly affected (see Figure 0-2A). These spectral changes may be due to a possible motion of the AgNPs and/or a change in the refractive index of the nanoplasmonic membrane facilitated by humidity across the experiment. We also spotted a blue shift of up to $20 \mathrm{~nm}$ in the experiments carried out with the other 10 volatile compounds and the absorbance peak was slightly increased or decreased (see Figure 0-2A). The origin of these spectral changes might be attributed to a reorganization of the AgNPs embedded in the nanocellulose triggered by humidity and the effect of the volatile compound on the nanopaper.


Figure 0-2. Specificity of the nanoplasmonic membrane as a corrosive vapor (ammonia) sensing platform. A. UV-Vis spectra of plasmonic nanopaper upon vapor exposure using an initial volume of $1000 \mu \mathrm{L}$ of different volatile compounds spontaneously volatilized for 18 hours. B. Blue shift observed in the UV-Vis spectra depending on the volatile compound exposed. C. Changes in optical density according to the volatile compound exposed. The error bars represent the standard deviation of three parallel experiments. a. Methanol. b. Ethanol c. Propanol. d. Acetic acid. e. Ethyl acetate. f. Acetonitrile. g. Ammonia. h. Acetone. i. Toluene. j. Indole. k. Water. 1. Triethylamine.

On the other hand, the color of the nanoplasmonic membranes exposed to the other volatile compounds has not been observed to change by the naked eye. As a consequence, we exploited the formula $\Delta \mathrm{OD}=1-\left(A / A_{0}\right)$ to analyze the absorbance peak modulation, where $\Delta \mathrm{OD}$ represents the changes in optical density; $A_{0}$ is the original intensity of the absorbance peak of the nanoplasmonic membrane; and $A$ is the final intensity of the absorbance peak of the studied nanoplasmonic membrane. The plasmonic nanopaper exposed to ammonia changed in color, which can be detected 
smoothly by the naked eye as described above. It also exhibited a blue shift of around $20 \mathrm{~nm}$ and, most importantly, its $\Delta \mathrm{OD}$ values were dramatically different, as can be noticed in Figure $0-2 \mathrm{~B}$ and C. These results prove that the explored phenomenon is specific to ammonia exposure. Moreover, AgNPs have been reported to undergo a continuous release of silver ions from their surface [28], which are known to react with ammonia forming the complex $\left[\mathrm{Ag}\left(\mathrm{NH}_{3}\right)_{2}\right]^{+}$. This complex can occur simultaneously with hydrogen bonding between the hydroxyl groups of nanocellulose and ammonia, which may boost the dissociation of the silver atoms and modulate the AgNP size. This mechanism is likely to contribute to the specificity of the explored sensing system.

\subsubsection{Sensing performance}

We explored several ammonia dose exposures for different times in order to determine the overall performance of the sensing principle using the experimental set-up described in Error! Reference source not found.B. Particularly, ammonia volumes from $10 \mu \mathrm{L}$ to $1000 \mu \mathrm{L}$, were spontaneously volatilized at room temperature for 2, 4, 8 and 12 hours. The corresponding initial evaporation rates were estimated as described in the ESI, and range from $c a .15 .7 \mathrm{ng} \mathrm{s}^{-1}$ to $c a .1200 \mathrm{ng} \mathrm{s}^{-1}$.

Figure 0-3A shows the UV-Vis spectra of the plasmonic nanopaper exposed to an initial ammonia volume of $10 \mu \mathrm{L}$ and $1000 \mu \mathrm{L}$ between 2 and 12 hours. The intensity of the UV-Vis absorbance was observed to be modulated as a function of ammonia dose and exposure time. The general behavior of this modulation was represented in terms of the $\triangle \mathrm{OD}$ values (obtained as discussed above); see Figure 0-3B, which displays a two-phase response with a sharp transition for the ammonia doses below an initial evaporation rate of $121 \mathrm{ng} \mathrm{s}^{-1}$ (corresponding to $50 \mu \mathrm{L}$ ) and a steady response for the ammonia doses with an initial evaporation rate between 176 and $1200 \mathrm{ng} \mathrm{s}^{-1}$ (100 and $1000 \mu \mathrm{L}$, respectively). $\Delta \mathrm{OD}$ values corresponding to the exposure to water were considered as blank values. Different limits of detection were estimated by interpolating the $\Delta \mathrm{OD}$ blank value plus eight times its standard deviation into the respective calibration curve, that is, according to the exposure time of the explored corrosive vapor (ammonia). 

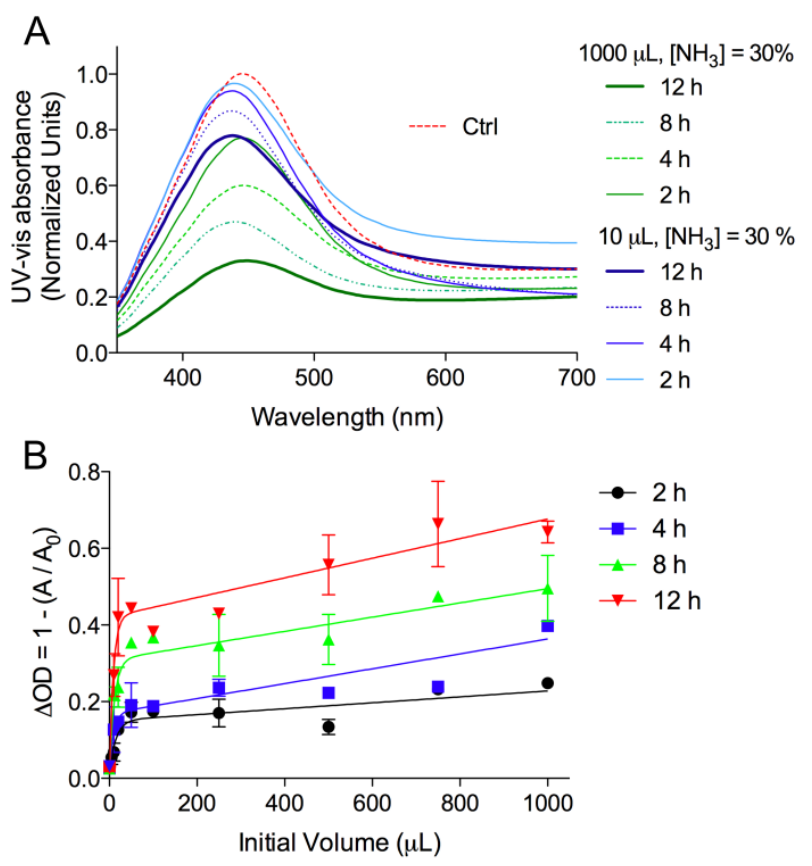

Figure 0-3. Behavior of the nanoplasmonic membrane as a corrosive vapor sensing platform. A. $\mathrm{UV}-\mathrm{Vis}$ spectra of the plasmonic nanopaper upon corrosive vapour (ammonia) exposure using 10 and $1000 \mu \mathrm{L}$ of $30 \%$ ammonia solution spontaneously volatilized for between 2 and 12 hours. B. Calibration curves displaying changes in optical density depending on the initial volume to be volatilized (from 10 to $1000 \mu \mathrm{L}$ ) and the exposure time (2-12 hours). The error bars represent the standard deviation of three parallel experiments.

Among the explored exposure times, the proposed sensing platform exhibited a limit of detection of $1515 \mu \mathrm{g}$ of ammonia spontaneously volatilized at room temperature during $2 \mathrm{~h}$ in a sealed $50 \mathrm{~mL}$ container (i.e., 30.3 ppmv). On the other hand, the explored sensing system displayed a limit of detection of $28.7 \mu \mathrm{g}$ of ammonia spontaneously volatilized at room temperature in a sealed $50 \mathrm{~mL}$ container for 8 hours (i.e., 0.574 ppmv), which was the lowest limit of detection observed. Table 0-1 summarizes the estimated limits of detection and their related initial evaporation rates (details on these estimations are discussed in the ESI). 
Table $0-1$. Limits of detection of the explored system $\underline{a}$

\begin{tabular}{lll}
\hline Exposure time (h) & LOD $^{\underline{b}}$ & $\operatorname{RIER}^{\boldsymbol{c}}\left(\mathbf{n g ~ s}^{-\mathbf{1}}\right)$ \\
\hline 2 & $\sim 30.3$ & $\sim 2.48$ \\
4 & $\sim 6.42$ & $\sim 0.51$ \\
8 & $\sim 0.574$ & $\sim 0.09$ \\
12 & & \\
\hline
\end{tabular}

${ }^{a}$ Details on these estimations are discussed in the ESI. ${ }^{b}$ Limit of detection. ${ }^{c}$ Related initial evaporation ratio. Food spoilage monitoring: a proof-of-concept

Aiming at demonstrating a practical application for this approach, as a proof-of-concept, we reasoned that the studied phenomenon could be useful for food spoilage monitoring given that ammonia is released as a major agent along with other volatile compounds during the degradation process, in particular trimethylamine, dimethylamine, histamine, putrescine, cadaverine, short-chain carbonyls, acids, sulfur compounds, and $\mathrm{N}$-cyclic compounds as well as unsaturated aldehydes in fish spoilage [29], and alcohols, aldehydes, esters, ketones, and carbon dioxide in meat spoilage [30].

We again used the experimental set-up illustrated in Error! Reference source not found.B. $5 \mathrm{~g}$ of fish or meat was placed inside the sealed container, and was left at room temperature for 60 hours. Unexpectedly, the original amber color of the nanoplasmonic membranes became grey for the fish spoilage monitoring and a taupe colour for the meat spoilage monitoring. Furthermore, the UV-Vis spectrum was dramatically modulated and the original peak absorbance disappeared (see Figure 0-4A and B). Interestingly, the size distribution of the AgNPs embedded in the nanocellulose was also dramatically modulated upon food spoilage exposure. As discussed above, the original size distribution bears an average particle diameter of around $10 \pm 7 \mathrm{~nm}$, whereas we ascertained that the average particle diameter upon food spoilage exposure was approximately $6 \pm 3 \mathrm{~nm}$ for fish spoilage monitoring, and $c a .6 \pm 5 \mathrm{~nm}$ for meat spoilage monitoring. 

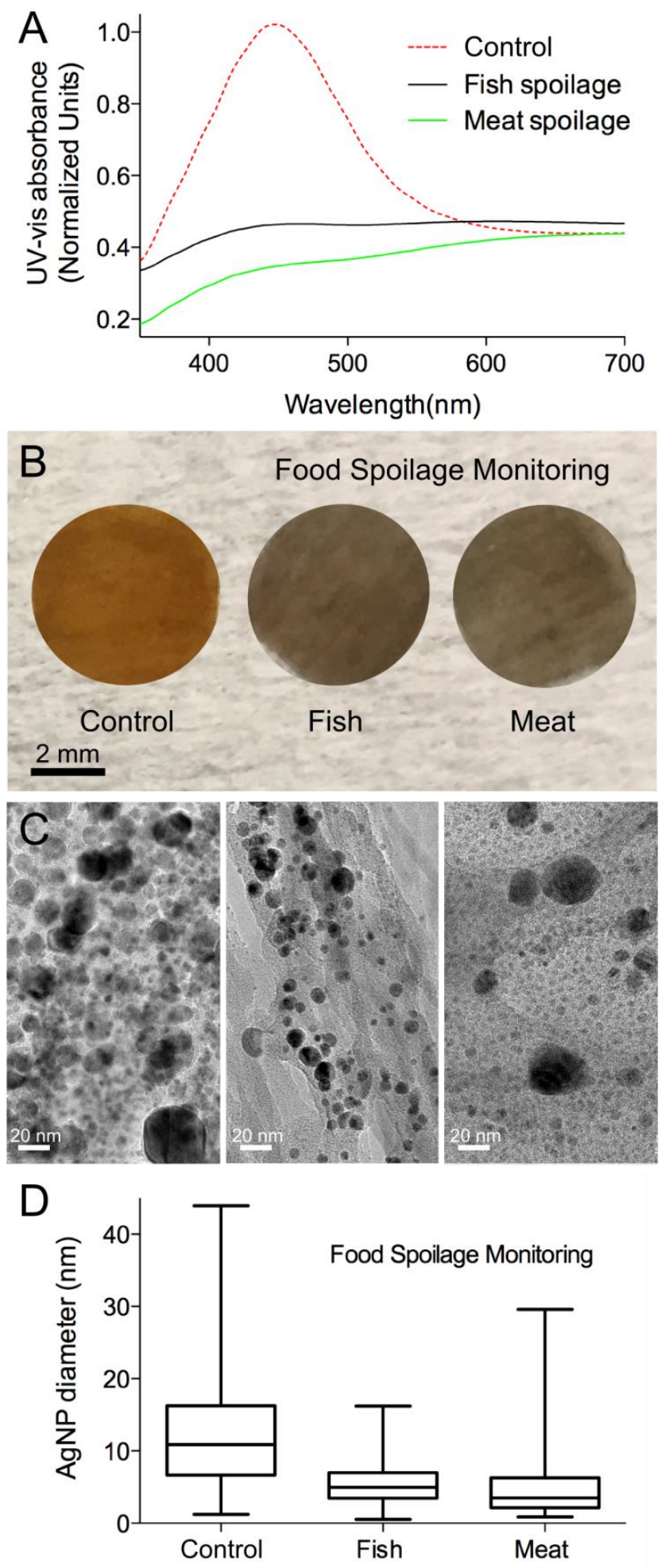

Figure 0-4. Food spoilage monitoring via plasmonic nanopaper. A. UV-Vis spectra of the nanoplasmonic membranes before food spoilage exposure (Ctrl) and after food spoilage exposure (ammonia and several volatile organic compounds are released during fish and meat 
spoilage). B. Appearance of the nanoplasmonic membranes before/after food spoilage monitoring. C. TEM micrographs, from left to right: control, fish spoilage and meat spoilage. D. Size distribution of the AgNPs embedded in the nanopaper before/after food spoilage monitoring, the box plots show the median, 25th and 75th percentiles and the extreme values of the respective size distributions (5-95 percentile).

On the other hand, we found that the distribution size of the embedded AgNPs ranged from 1 to 16 $\mathrm{nm}$ upon fish spoilage exposure, and from 1 to $30 \mathrm{~nm}$ upon meat spoilage exposure. However, the population density of the embedded AgNPs was not dramatically affected during food spoilage monitoring. Thus, we concluded that the volatile compounds released during food spoilage effectively modulated the size of the embedded AgNPs and consequently the plasmonic properties of the sensing platform. Figure 0-4C displays the corresponding TEM micrographs and Figure 0-4D plots the estimated size distributions. Table 0-2 summarizes our study on the modulation of the population density and size distribution of the AgNPs embedded in the nanopaper via volatile compound exposure.

Table 0-2. Modulation of population density (PD) and average size (AS) of the AgNPs embedded in the nanopaper via volatile compound exposure.

\begin{tabular}{llll}
\hline \hline & \multicolumn{2}{l}{ PD $^{\underline{a}}($ AgNP per AgNP- } & Resulting \\
Exposure & $\left.\boldsymbol{\mu m}^{2}\right)$ & $\mathbf{A S}^{\boldsymbol{a}}(\mathbf{n m})$ & colour \\
\hline Control & $\sim 1473 \pm 227$ & $\sim 10 \pm 7$ & Amber \\
Ammonia & $\sim 302 \pm 38$ & $\sim 17 \pm 12$ & Light amber \\
Fish & $\sim 1213 \pm 251$ & $\sim 6 \pm 3$ & Grey \\
spoilage & & & \\
Meat & $\sim 1376 \pm 244$ & $\sim 6 \pm 5$ & Taupe \\
spoilage & & &
\end{tabular}

${ }^{a}$ Three TEM micrographs corresponding to each type of exposure were analyzed to estimate these values. 


\subsubsection{Raman analysis}

Additionally, we performed a Raman analysis of the explored materials. In particular, we observed that the employed bare bacterial cellulose displays its main Raman signature from 900 to 1500 $\mathrm{cm}^{-1}$ and from 2700 to $3500 \mathrm{~cm}^{-1}$, with the most prominent peaks being those centred at $\sim 2900$ and $\sim 3375 \mathrm{~cm}^{-1}$. Moreover, the peak centred at $\sim 3375 \mathrm{~cm}^{-1}$ disappears after synthesizing the AgNPs using the nanocellulose as a reducing agent, and a new set of peaks rise from 1500 to $1700 \mathrm{~cm}^{-1}$ due to the presence of the AgNPs. Interestingly, the Raman signature of the nanoplasmonic membrane around $1350 \pm 450 \mathrm{~cm}^{-1}$ was modulated upon ammonia exposure, whereas the peaks centred at 2900 and $3375 \mathrm{~cm}^{-1}$ were similar to those of bare nanocellulose. Likewise, the Raman fingerprint of the nanoplasmonic membrane was modulated upon fish/meat spoilage, with those prominent peaks centred at $\sim 2900 \mathrm{~cm}^{-1}$ and $\sim 3375 \mathrm{~cm}^{-1}$ being renewed, which can be ascribed to the partial or complete etching of the embedded AgNPs and can be considered further evidence of the studied phenomena (see Figure 0-5).

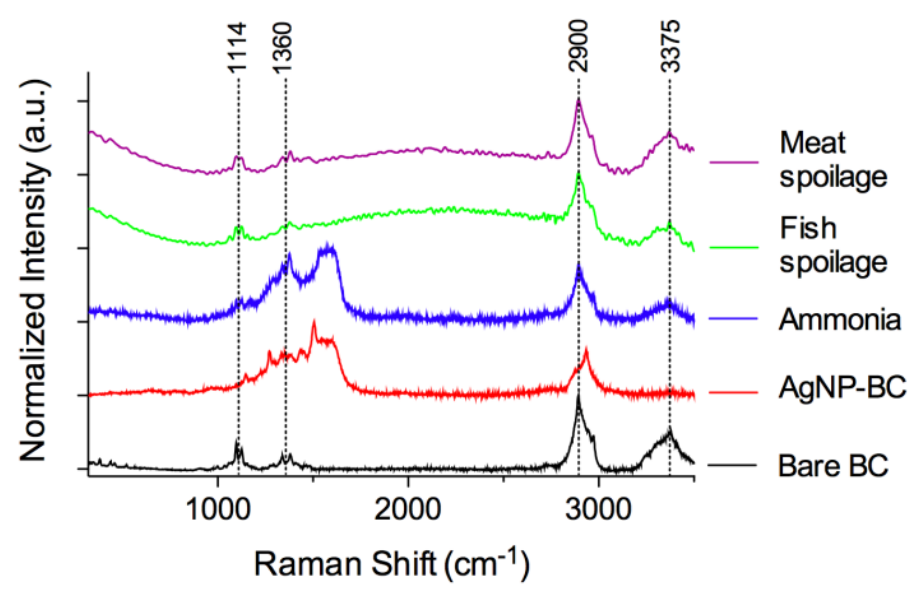

Figure 0-5. Raman signature of the explored materials: bare bacterial cellulose (BC), bacterial cellulose decorated with silver nanoparticles (AgNP-BC) and AgNP-BC exposed to ammonia and fish/meat spoilage.

\subsection{Conclusions}

We discovered that the population density and size of silver nanoparticles embedded within bacterial cellulose nanopaper can be easily modulated via corrosive vapor (ammonia) exposure even in a mixture of volatile compounds released during food spoilage. In fact, AgNPs embedded in nanopaper 
were partially or completely etched by the explored corrosive vapor exposure, modulating the plasmonic properties of the studied plasmonic nanopaper. As a consequence, the nanoplasmonic membrane changed in color from amber to light amber upon ammonia exposure and from amber to grey or taupe in colour upon fish or meat spoilage exposure, respectively. These phenomena are proposed for a straightforward volatile compound sensing platform principle that can be applied for visual detection using a flexible, transparent, permeable and stable single-use nanoplasmonic membrane. This approach opens the way to new possibilities and capabilities for smart packaging and gas sensing-related applications.

\subsection{Acknowledgements}

This work was supported by MINECO (Spain, MAT2014-52485-P). ICN2 acknowledges support from the Severo Ochoa Program (MINECO, Grant SEV-2013-0295). Nanobiosensors and Bioelectronics Group acknowledges the support from Secretaria d'Universitats i Recerca del Departament d'Economia i Coneixement de la Generalitat de Catalunya (2014 SGR 260). B. H. thanks the FRQNT (Quebec, Canada), NSERC (Canada) and the industrial partners of 3SPack Chair Saputo and Prolamina for funding her research stay in Spain. The authors are grateful to Dr. Hossein Yousefi for providing the bacterial cellulose utilized in this research.

\subsection{References}

[1] H. Goesmann and C. Feldmann, "Nanoparticulate functional materials," Angewandte Chemie International Edition, vol. 49, pp. 1362-1395, 2010.

[2] X. Chen, S. G. Parker, G. Zou, W. Su, and Q. Zhang, " $\beta$-Cyclodextrin-functionalized silver nanoparticles for the naked eye detection of aromatic isomers," ACS nano, vol. 4, pp. 6387-6394, 2010.

[3] P. D. Howes, R. Chandrawati, and M. M. Stevens, "Colloidal nanoparticles as advanced biological sensors," Science, vol. 346, p. 1247390, 2014.

[4] J. N. Anker, W. P. Hall, O. Lyandres, N. C. Shah, J. Zhao, and R. P. Van Duyne, "Biosensing with plasmonic nanosensors," Nature materials, vol. 7, pp. 442-453, 2008. 
[5] M.-P. N. Bui, S. Ahmed, and A. Abbas, "Single-Digit Pathogen and Attomolar Detection with the Naked Eye Using Liposome-Amplified Plasmonic Immunoassay," Nano Letters, vol. 15, pp. 6239-6246, 2015/09/09 2015.

[6] T. Xu, H. Shi, Y.-K. Wu, A. F. Kaplan, J. G. Ok, and L. J. Guo, "Structural Colors: From Plasmonic to Carbon Nanostructures," Small, vol. 7, pp. 3128-3136, 2011.

[7] M. I. Stockman, "Nanoplasmonic sensing and detection," Science, vol. 348, pp. 287-288, 2015.

[8] E. Busseron, Y. Ruff, E. Moulin, and N. Giuseppone, "Supramolecular self-assemblies as functional nanomaterials," Nanoscale, vol. 5, pp. 7098-7140, 2013.

[9] S. Szunerits and R. Boukherroub, "Sensing using localised surface plasmon resonance sensors," Chemical Communications, vol. 48, pp. 8999-9010, 2012.

[10] S. Su, X. Zuo, D. Pan, H. Pei, L. Wang, C. Fan, et al., "Design and applications of gold nanoparticle conjugates by exploiting biomolecule-gold nanoparticle interactions," Nanoscale, vol. 5, pp. 2589-2599, 2013.

[11] E. Morales-Narváez, H. Golmohammadi, T. Naghdi, H. Yousefi, U. Kostiv, D. Horák, et al., "Nanopaper as an Optical Sensing Platform," ACS nano, vol. 9, pp. 7296-7305, 2015.

[12] D. Fine, F. Rufeh, and D. Lieb, "Group analysis of volatile and non-volatile N-nitroso compounds," Nature, vol. 247, pp. 309-310, 1974.

[13] K. D. van de Kant, L. J. van der Sande, Q. Jöbsis, O. C. van Schayck, and E. Dompeling, "Clinical use of exhaled volatile organic compounds in pulmonary diseases: a systematic review," Respiratory Research, vol. 13, p. 117, December 212012.

[14] B. H. King, A. Gramada, J. R. Link, and M. J. Sailor, "Internally Referenced Ammonia Sensor Based on an Electrochemically Prepared Porous SiO2 Photonic Crystal," Advanced Materials, vol. 19, pp. 4044-4048, 2007.

[15] T. A. Kumar, E. Capua, M. Tkachev, S. N. Adler, and R. Naaman, "Hybrid OrganicInorganic Biosensor for Ammonia Operating under Harsh Physiological Conditions," Advanced Functional Materials, vol. 24, pp. 5833-5840, 2014. 
[16] E. Tait, J. D. Perry, S. P. Stanforth, and J. R. Dean, "Identification of Volatile Organic Compounds Produced by Bacteria Using HS-SPME-GC-MS," Journal of Chromatographic Science, vol. 52, pp. 363-373, 2014.

[17] Y. Xu, W. Cheung, C. L. Winder, and R. Goodacre, "VOC-based metabolic profiling for food spoilage detection with the application to detecting Salmonella typhimuriumcontaminated pork," Analytical and Bioanalytical Chemistry, vol. 397, pp. 2439-2449, July 012010.

[18] M. Hakim, Y. Y. Broza, O. Barash, N. Peled, M. Phillips, A. Amann, et al., "Volatile organic compounds of lung cancer and possible biochemical pathways," Chemical reviews, vol. 112, pp. 5949-5966, 2012.

[19] "http://www.environmental-expert.com/products/ammonia-test-paper-301704, 2015."

[20] http://www.ctlscientific.com/cgi/display.cgi?item_num=90722.

[21] N. Pourreza, H. Golmohammadi, T. Naghdi, and H. Yousefi, "Green in-situ synthesized silver nanoparticles embedded in bacterial cellulose nanopaper as a bionanocomposite plasmonic sensor," Biosensors and Bioelectronics, vol. 74, pp. 353-359, 2015.

[22] H. Zhu, S. Parvinian, C. Preston, O. Vaaland, Z. Ruan, and L. Hu, "Transparent nanopaper with tailored optical properties," Nanoscale, vol. 5, pp. 3787-3792, 2013.

[23] M.-C. Hsieh, C. Kim, M. Nogi, and K. Suganuma, "Electrically conductive lines on cellulose nanopaper for flexible electrical devices," Nanoscale, vol. 5, pp. 9289-9295, 2013.

[24] D. Klemm, F. Kramer, S. Moritz, T. Lindström, M. Ankerfors, D. Gray, et al., "Nanocelluloses: A New Family of Nature-Based Materials," Angewandte Chemie International Edition, vol. 50, pp. 5438-5466, 2011.

[25] D. Klemm, B. Heublein, H.-P. Fink, and A. Bohn, "Cellulose: Fascinating Biopolymer and Sustainable Raw Material," Angewandte Chemie International Edition, vol. 44, pp. 3358-3393, 2005. 
[26] W. Kang, C. Yan, C. Y. Foo, and P. S. Lee, "Foldable Electrochromics Enabled by Nanopaper Transfer Method," Advanced Functional Materials, vol. 25, pp. 4203-4210, 2015.

[27] L. R. Narasimhan, W. Goodman, and C. K. N. Patel, "Correlation of breath ammonia with blood urea nitrogen and creatinine during hemodialysis," Proceedings of the National Academy of Sciences, vol. 98, pp. 4617-4621, April 10, 20012001.

[28] J. Liu, D. A. Sonshine, S. Shervani, and R. H. Hurt, "Controlled Release of Biologically Active Silver from Nanosilver Surfaces," ACS Nano, vol. 4, pp. 6903-6913, 2010/11/23 2010.

[29] M. K. Morsy, K. Zór, N. Kostesha, T. S. Alstrøm, A. Heiskanen, H. El-Tanahi, et al., "Development and validation of a colorimetric sensor array for fish spoilage monitoring," Food Control, vol. 60, pp. 346-352, 2016/02/01/ 2016.

[30] B. Zhang, S. Ye, G. Xiao, and D. Dong, "Identification of beef spoilage via the analysis of volatiles using long optical-path Fourier transform infrared spectroscopy," Analytical Methods, vol. 7, pp. 5891-5897, 2015. 


\title{
1.8 Supporting Information
}

Modulation of population density and size of silver nanoparticles embedded in bacterial cellulose via ammonia exposure: Visual detection of volatile compounds in a piece of plasmonic nanopaper

\author{
B. Heli ${ }_{a b}$, E. Morales-Narváez ${ }^{a}$, H. Golmohammadi $c$, A. Ajji ${ }^{*_{b}}$ and A. Merkoçi ${ }^{*_{d}}$ \\ ¿Catalan Institute of Nanoscience and Nanotechnology (ICN2), CSIC and The Barcelona Institute of \\ Science and Technology, Campus UAB, Bellaterra, Barcelona 08193, Spain \\ '3SPack, CREPEC, Département de génie chimique, Polytechnique Montréal, Montréal, Québec, \\ Canada \\ 'ACECR-Production Technology Research Institute, Ahvaz, 6139684689, Iran \\ ICREA - Catalan Institution for Research and Advanced Studies, Barcelona, 08010, Spain.
}

\subsubsection{Supporting information content}

1. Supporting Figures

2. Estimation of Evaporation Rates and Limits of Detection

4. Supplementary References 


\subsubsection{Figures}
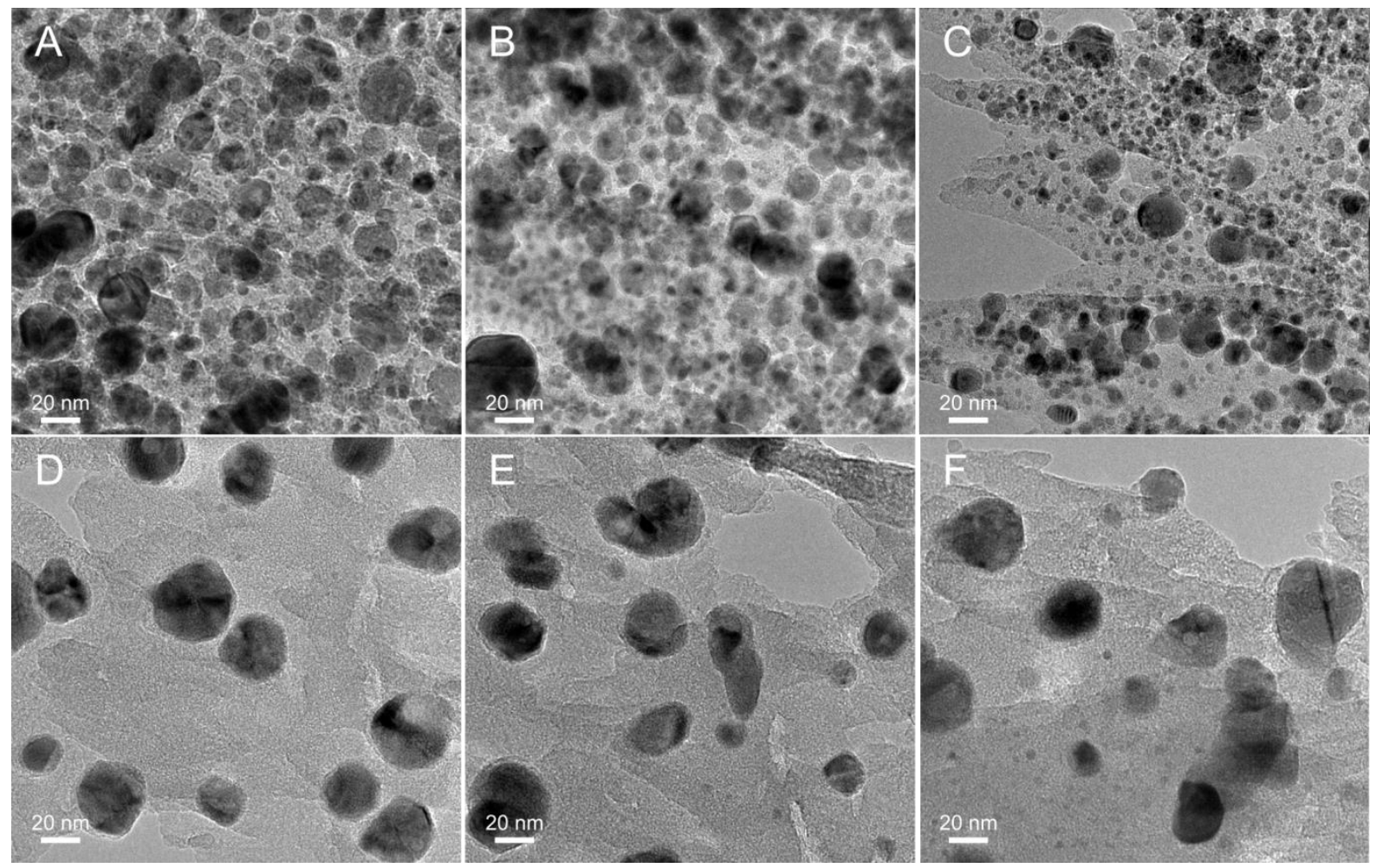

Figure S0-1. TEM micrographs showing the population density of AgNPs embedded in BC without $\mathrm{NH} 3$ vapor exposure (A-C) and after NH3 vapor exposure (D-F). The population density of AgNPs at the foreground in images A-C has been estimated to be $1473 \pm 227 \mathrm{AgNP}_{\mu} \mathrm{m}^{-2}$, whereas the population density of AgNPs at the foreground in images D-F is around $302 \pm 38$ AgNP $\mu \mathrm{m}^{-2}$. AgNP-BC in images D-F was exposed at an initial vapor rate of around $\sim 1.2 \mu \mathrm{g} \mathrm{s}^{-} 1$ for 12 hours. TEM micrographs were analyzed via image processing through ImageJ $1.48 \mathrm{v}$ (Wayne Rasband, National Institutes of Health, Bethesda, MD) in order to estimate the population density of nanoparticles embedded in the BC-based composite. 

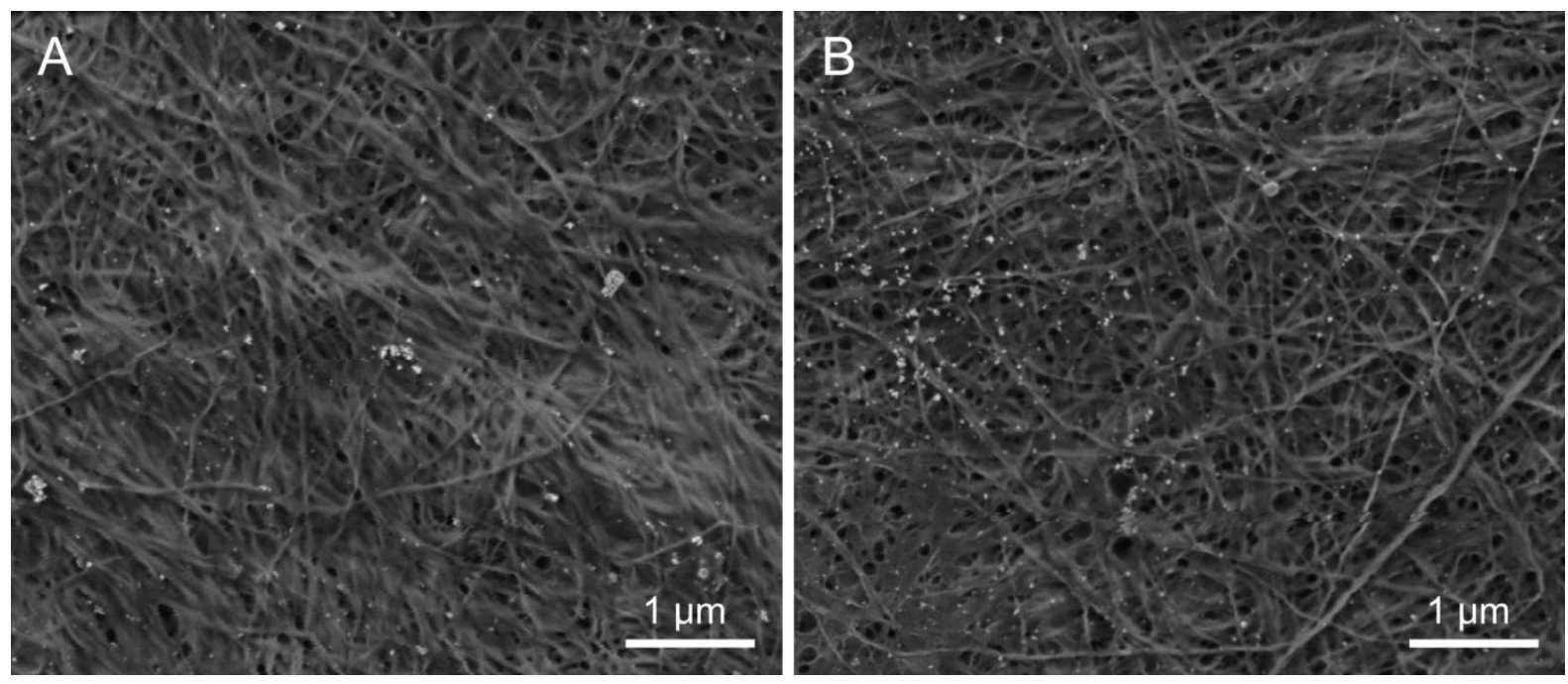

Figure S0-2. SEM micrographs of AgNP-BC without NH3 vapor exposure (A) and after NH3 vapor exposure (B). No damage or structural changes were observed in the nanofibers.

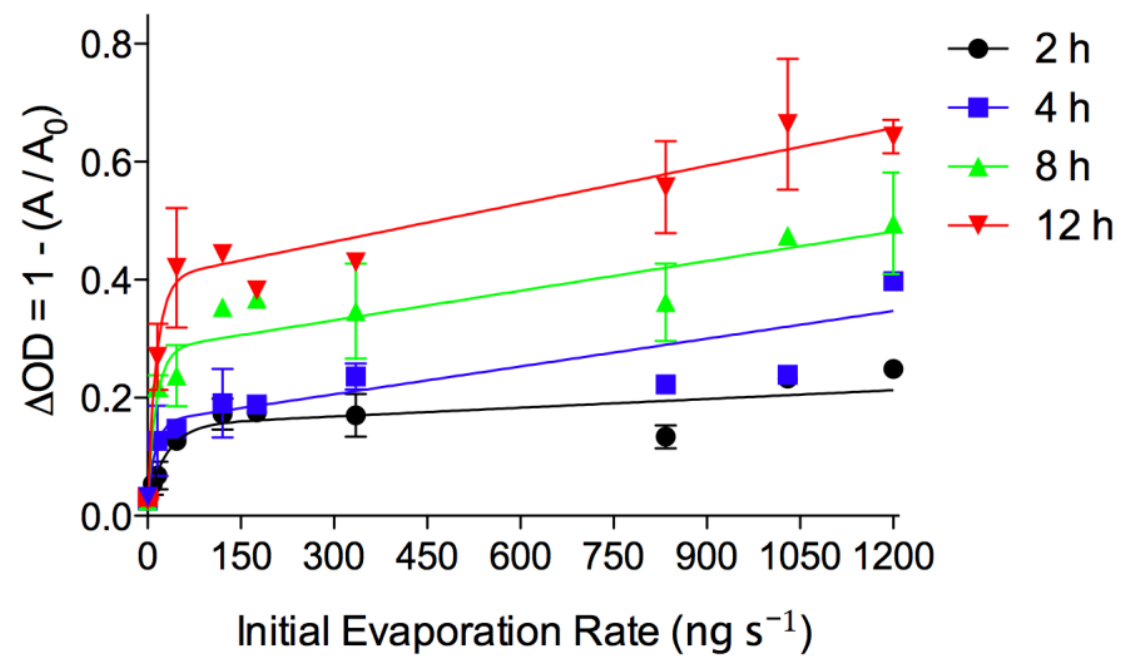

Figure S0-3. Calibration curves displaying changes in optical density depending on the initial evaporation rate and the exposure time. The error bars represent the standard deviation of three parallel experiments. 


\subsubsection{Estimation of Evaporation rates and Limits of Detection}

Estimation of Evaporation rates and Limits of Detection The evaporation rate was estimated via the computations suggested by the National Ocean Service, (2013, National Oceanic and Atmospheric Administration, http://goo.gl/ebOf2T), which are based on the model proposed by Kawamura and Mackay [1]. This estimation was carried out considering a negligible wind speed

of $1 \mu \mathrm{m} \mathrm{s}^{-1}$, an ammonia concentration of $30 \%$, an ambient temperature of $25^{\circ} \mathrm{C}$ and measuring the dimensions of the drops belonging to the respective studied volumes after being deposited onto the bottom of the kind of container employed throughout this research, see details in Table S1 below.

Table S 0-1. Estimation of initial evaporation rate of $\mathrm{NH}_{3}$.

\begin{tabular}{|c|c|c|c|c|}
\hline \multirow{2}{*}{$\begin{array}{l}\text { Volume } \\
(\mu \mathrm{L})\end{array}$} & \multicolumn{2}{|c|}{ Dimensions of the drop } & \multicolumn{2}{|c|}{ Evaporation rate } \\
\hline & Length $(\mathrm{cm})$ & Width $(\mathrm{cm})$ & $\left(\mathrm{ng} \mathrm{s}^{-1}\right)$ & $\left(\mu \mathrm{g} \mathrm{h}^{-1}\right)$ \\
\hline 5 & $0.21 \pm 0.11$ & $0.21 \pm 0.01$ & 8.51 & 30.6 \\
\hline 10 & $0.29 \pm 0.01$ & $0.29 \pm 0.01$ & 15.7 & 56.5 \\
\hline 20 & $0.54 \pm 0.05$ & $0.5 \pm 0.05$ & 46.9 & 168.8 \\
\hline 50 & $0.88 \pm 0.10$ & $0.83 \pm 0.07$ & 121.0 & 435.6 \\
\hline 100 & $1.15 \pm 0.05$ & $0.96 \pm 0.02$ & 176.0 & 633.6 \\
\hline 250 & $1.61 \pm 0.05$ & $1.35 \pm 0.05$ & 335.0 & 1206.0 \\
\hline 500 & $2.96 \pm 0.15$ & $1.96 \pm 0.05$ & 834.0 & 3002.4 \\
\hline 750 & $3.03 \pm 0.05$ & $2.36 \pm 0.11$ & 1030.0 & 3708.0 \\
\hline 1000 & $3.1 \pm 0.00$ & $2.71 \pm 0.10$ & 1200.0 & 4320.0 \\
\hline
\end{tabular}

We utilized the formula $\Delta \mathrm{OD}=1-\left(\mathrm{A} / \mathrm{A}_{0}\right)$ to analyze the absorbance peak modulation, where $\Delta \mathrm{OD}$ represents the changes in optical density; $\mathrm{A}_{0}$, the original intensity of the absorbance peak 
of the nanoplasmonic membrane; and A the final intensity of the absorbance peak of the nanoplasmonic membrane. $\triangle \mathrm{OD}$ values corresponding to the exposure to water were considered as blank values. Different limits of detection were estimated by interpolating the $\Delta$ OD blank value plus eight times its standard deviation into the respective calibration curve, that is, according to the exposure time of the explored corrosive vapor.

These resulting interpolations are expressed in terms of $\mu \mathrm{L}$. As the analyzed ammonia (30\%) has

a density of $897 \mu \mathrm{g} \mu \mathrm{L}^{-1}$, the mass corresponding to these calculated values was estimated using this density value as a conversion factor. The limits of detection in terms of initial evaporation rate $\left(\mathrm{ng} \mathrm{s}^{-1}\right.$ ) were obtained by interpolating the respective $\Delta \mathrm{OD}$ values using the curves plotted in Figure S0-3.

Author Contributions A. M. and E. M-N. conceived the overall concept. E. M-N., B. H. and H. G. designed the experiments. E. M-N., B. H., H. G. and A. M. analyzed the data. B. H. performed the experiments. E. M-N., wrote the manuscript with input of B.H., A. A. and A. M. E. M-N. performed the figures with input of B.H. A. M. and E. M-N. supervised the overall project.

\subsubsection{Supplementary References}

[1] P. I. Kawamura and D. Mackay, "The evaporation of volatile liquids," Journal of hazardous Materials, vol. 15, pp. 343-364, 1987. 